\title{
Análise epidemiológica da segunda onda de COVID-19 no estado da Bahia
}

\author{
Epidemiological analysis of the second wave of COVID-19 in the state of Bahia \\ Análisis epidemiológico de la segunda ola de COVID-19 en el estado de Bahía
}

Lucas Reis Oliveira $^{1 *}$, Anna Sophia Almeida Gouveia ${ }^{2}$, Daniel Andrade Matias ${ }^{3}$, Wianne Santos Silva ${ }^{4}$, Vitória Dias dos Santos ${ }^{4}$, Marlon Rubini Toazza ${ }^{2}$.

\section{RESUMO}

Objetivo: Analisar a evolução do número de casos, óbitos e ocupação de leitos de UTI por COVID-19 no estado da Bahia durante a segunda onda. Métodos: Trata-se de um estudo epidemiológico, descritivo e transversal, no qual foram analisados os casos de COVID-19 no estado da Bahia notificados e publicados nos boletins epidemiológicos da Secretaria Estadual de Saúde da Bahia em janeiro e fevereiro de 2021. Resultados: A Bahia apresentou durante o período de análise do estudo 190.637 casos de COVID-19, com 2.690 óbitos no mesmo período. Observou-se, também, que houve uma média de ocupação de 776 leitos de UTI no período e local supracitados. O mês de fevereiro obteve maiores taxas de casos, óbitos e ocupação de leitos de UTI. A faixa etária com maior número de casos foi a dos indivíduos entre 30 e 39 anos, enquanto os idosos com 80 ou mais anos foram os que mais vieram a óbito proporcionalmente. Também podemos observar que indivíduos com comorbidades apresentaram piores desfechos em relação aos indivíduos sem comorbidades. Conclusão: Observou-se aumento de casos, óbitos e demanda por leitos de UTI, bem como que pacientes mais idosos e com comorbidades possuíram piores desfechos.

Palavras-chave: COVID-19, Epidemiologia, Brasil.

\section{ABSTRACT}

Objective: To analyze the evolution of case numbers, deaths and occupancy of ICU beds due to COVID-19 in the state of Bahia during the second wave. Methods: This is an epidemiological, descriptive and cross-sectional study, which were analyzed COVID-19 cases in the state of Bahia notified and published in the epidemiological bulletin of Bahia's State Department of Health in January and February 2021. Results: During the analysis period of the study, Bahia presented 190,637 cases of COVID-19, with 2,690 deaths in the same period. It was also noticed an average occupancy of 776 ICU beds in the mentioned period and place. February had higher rates of cases, deaths and occupancy of ICU beds. The age group with the largest number of cases was individuals between 30 and 39 years old, while the elderly aged 80 or more were the ones who proportionally died the most. We can also observe that individuals with comorbidities had worse outcomes compared to individuals without comorbidities. Conclusion: It was noticed a increase in number of cases, deaths and occupancy of ICU beds, as well as that older patients with comorbidities had worse outcomes.

Keywords: COVID-19, Epidemiology, Brazil.

\section{RESUMEN}

Obietivo: Analizar la evolución del número de casos, defunciones y ocupación de camas de UCI por COVID-19 en el estado de Bahía durante la sequnda ola. Métodos: Se trata de un estudio epidemiológico, descriptivo y transversal, en el que se analizaron los casos de COVID-19 en el estado de Bahía que fueron notificados y publicados en el boletín epidemiolóaico de la Secretaría de Salud del Estado de Bahía en enero v febrero de 2021. Resultados: Bahía presentó 190.637 casos de COVID-19 durante el período de análisis del estudio, con 2.690 muertes en el mismo período. También hubo una ocupación promedio de 776 camas de UCl en el período y lugar antes mencionado. El mes de febrero tuvo mayores tasas de casos, muertes y ocupación de camas de UCl. El grupo de edad con mayor número de casos fue el de individuos entre 30 y 39 años, mientras que los ancianos de 80 o más años fueron los que proporcionalmente más fallecieron. También podemos observar que los individuos con comorbilidades tuvieron peores resultados en comparación con los individuos sin comorbilidades. Conclusión: Hubo un aumento de casos, muertes y demanda de camas de UCI, así como que los pacientes mayores con comorbilidades tuvieron peores resultados.

Palabras clave: COVID-19, Epidemiología, Brasil.

${ }^{1}$ Universidade Federal de Sergipe (UFS), Lagarto - SE. *E-mail: lucas.reis2016@hotmail.com

2 Universidade de Santa Cruz do Sul (UNISC), Santa Cruz do Sul - RS.

3 Universidade Federal de Sergipe (UFS), Aracaju - SE.

${ }^{4}$ Universidade Tiradentes (UNIT), Aracaju - SE. 


\section{INTRODUÇÃO}

Um surto de casos de pneumonia viral em Wuhan, província de Hubei, centro da China, foi notificado pela primeira vez à Organização Mundial da Saúde (OMS) em 31 de dezembro de 2019 (WORLD HEALTH ORGANIZATION, 2020a). Posteriormente, o novo vírus foi agrupado na família dos coronavírus, e sua manifestação clínica extrema foi denominada de síndrome respiratória aguda grave por coronavírus-2 (SARS-CoV-2), causada pela doença coronavírus 2019 (COVID-19) (LU R, et al., 2020).

Desde então o SARS-CoV-2 se propagou mundialmente: em um curto período a situação se agravou a tal ponto que, em março de 2020, se estabeleceu um estado de pandemia (WU X, et al., 2020). Embora o cenário pandêmico atual tenha sido completamente inesperado, os coronavírus não eram desconhecidos pela comunidade científica atual posto que, nos últimos 20 anos, causaram duas epidemias em grande escala: a síndrome respiratória aguda grave (SARS), surgida em 2002 e a qual entre 2003 e 2004 já havia sido controlada, e a síndrome respiratória do Oriente Médio (MERS), a qual eclodiu em 2012 e até os dias atuais ainda apresenta casos, no entanto em uma escala muito reduzida (DROSTEN C, et al., 2003; WALLACE A, 2020).

O mecanismo de patogênese do SARS-CoV-2 é semelhante ao dos demais representantes da família dos coronavírus. A infecção ocorre através da invasão das células do hospedeiro por meio da enzima conversora de angiotensina 2 (ECA2), que atua como receptor do patógeno (ZHOU P, et al., 2020). Dessa forma, a gravidade da infecção está intrinsecamente relacionada à expressão da ECA2 e capacidade de ligação viral à enzima, à medida que a distribuição da ECA2 nos tecidos mostra correlação com os locais de infecção e a patologia da doença (KAI H e KAI M, 2020; TURNER AJ, et al., 2004).

Além disso, o vírus tem a capacidade de atacar outros órgãos que também expressam a ECA2, como o coração, sistema renal e trato gastrointestinal (BENNARDO F, et al., 2020; ROSE-JOHN S, 2018; CHEN C, et al., 2020). Consequentemente, além dos sinais e sintomas respiratórios típicos, sintomas digestivos e lesões hepáticas têm sido relatados durante o curso da doença. Assim, a sintomatologia extrapulmonar pode ser, em alguns pacientes, apenas a queixa inicial (DI GENNARO F, et al., 2020; LEE I, et al., 2020; GUAN W, et al., 2020).

Contudo, ainda que apresentem semelhanças, há uma diferença significativa observada no isolamento de vírus vivos de esfregaços de nasofaringe e rinofaringe entre o SARS-CoV-1 e o SARS-CoV-2, sugerindo que a replicação viral no tecido respiratório superior é ativa e que o SARS-CoV-2 é mais eficiente no que tange a disseminação através da eliminação ativa de vírus faríngeos (WÖLFEL R, et al., 2020). Assim, a transmissibilidade do novo coronavírus é potencialmente maior e, por conseguinte, seu principal modo de propagação é por meio de gotículas e aerossóis em alta concentração produzidos nas vias aéreas. Tal fato significa, portanto, que as medidas de proteção, como máscaras e higienização das mãos, podem ser efetivas na prevenção da infecção (WORLD HEALTH ORGANIZATION, 2020b).

As características fisiopatológicas da Covid-19 grave ocorrem em virtude de um processo pneumônico agudo com infiltrados inflamatórios teciduais e trombose microvascular que cursa com extensa opacidade radiológica (CARSANA L, et al., 2020). Os sintomas clínicos típicos dos pacientes acometidos são febre, cefaleia, tosse seca e dispneia. Os achados laboratoriais mais comuns são linfopenia e níveis levemente elevados de aminotransferases. Desse modo, a doença pode resultar em morte devido à insuficiência respiratória progressiva em decorrência de dano alveolar (DROSTEN C, et al., 2003).

Diante desse contexto e considerando o número exorbitante de casos de COVID-19, o presente estudo busca analisar a evolução do número de casos, óbitos e ocupação de leitos de UTI por COVID-19 no estado da Bahia durante a segunda onda, apresentando dados epidemiológicos sobre os casos e óbitos notificados no estado da Bahia nos 2 primeiros meses de 2021.

\section{MÉTODOS}

Trata-se de um estudo epidemiológico, descritivo e transversal, no qual foram analisados os casos de COVID-19 no estado da Bahia entre os dias 01/01/2021 e 28/02/2021. 
O estado da Bahia é o mais populoso da região nordeste e o quarto mais populoso do país, com mais de 14 milhões de habitantes. A Bahia possui uma área territorial de $564.760,427 \mathrm{~km}^{2}$ dividida em 417 municípios. O estado é o maior do nordeste e ocupa a quinta colocação no país em extensão territorial (IBGE, 2021).

No que diz respeito à saúde, o estado é dividido em nove núcleos regionais de saúde: centro-leste, centro-norte, extremo-sul, leste, nordeste, norte, oeste, sudoeste, sul. Esses nove núcleos regionais comportam as 28 regionais de saúde (Alagoinhas, Barreiras, Brumado, Camaçari, Cruz das Almas, Feira de Santana, Guanambi, Ibotirama, Ilhéus, Irecê, Itaberaba, Itabuna, Itapetinga, Jacobina, Jequié, Juazeiro, Paulo Afonso, Porto Seguro, Ribeira do Pombal, Salvador, Santa Maria da Vitória, Santo Antônio de Jesus, Seabra, Senhor do Bonfim, Serrinha, Teixeira de Freitas, Valença e Vitória da Conquista) (SESAB, 2021a; SESAB, 2021b).

A presente pesquisa foi realizada por meio da tabulação e análise de dados extraídos dos boletins epidemiológicos diários divulgados pela Secretaria de Saúde da Bahia (SESAB). A população envolvida na amostra diz respeito aos indivíduos que foram confirmados como casos e/ou óbitos durante os dias 01 de janeiro de 2021 e 28 de fevereiro de 2021. Os dados foram tabulados no programa Microsoft Office Excel, 0 qual foi a base para cálculo de medidas de tendência central.

Ademais, em consonância com a Lei № 12.527 de 18 de novembro de 2011 e com a Resolução № 510 , de 7 de abril de 2016, o presente estudo dispensou submissão ao Comitê de Ética em Pesquisa e, também, de Termo de Consentimento Livre e Esclarecido, uma vez que foram utilizados dados epidemiológicos de um domínio público e que não apresenta quaisquer informações que identifiquem os indivíduos (BRASIL, 2011; BRASIL, 2016).

\section{RESULTADOS}

No período compreendido entre 01 de janeiro de 2021 e 28 de fevereiro de 2021, houve 190.637 casos de COVID-19 no estado da Bahia. O dia 02 de janeiro foi o que apresentou a menor quantidade de casos confirmados (602), enquanto o dia 27 de fevereiro obteve o maior número, com 6.520 casos confirmados. 0 período supracitado apresentou uma média de 3.231 casos. O Gráfico 1 apresenta a evolução diária de casos durante o período. O total de leitos de UTI ocupados no período variou entre 700 (dia 29 de janeiro de 2021) e 983 (dia 28 de fevereiro de 2021), com uma média de 776 leitos ocupados por dia no período. 0 Gráfico 2, por sua vez, apresenta a evolução diária de ocupação de leitos de UTI no estado no período supracitado.

Gráfico 1 - Número de casos de COVID-19 confirmados por dia no estado da Bahia.

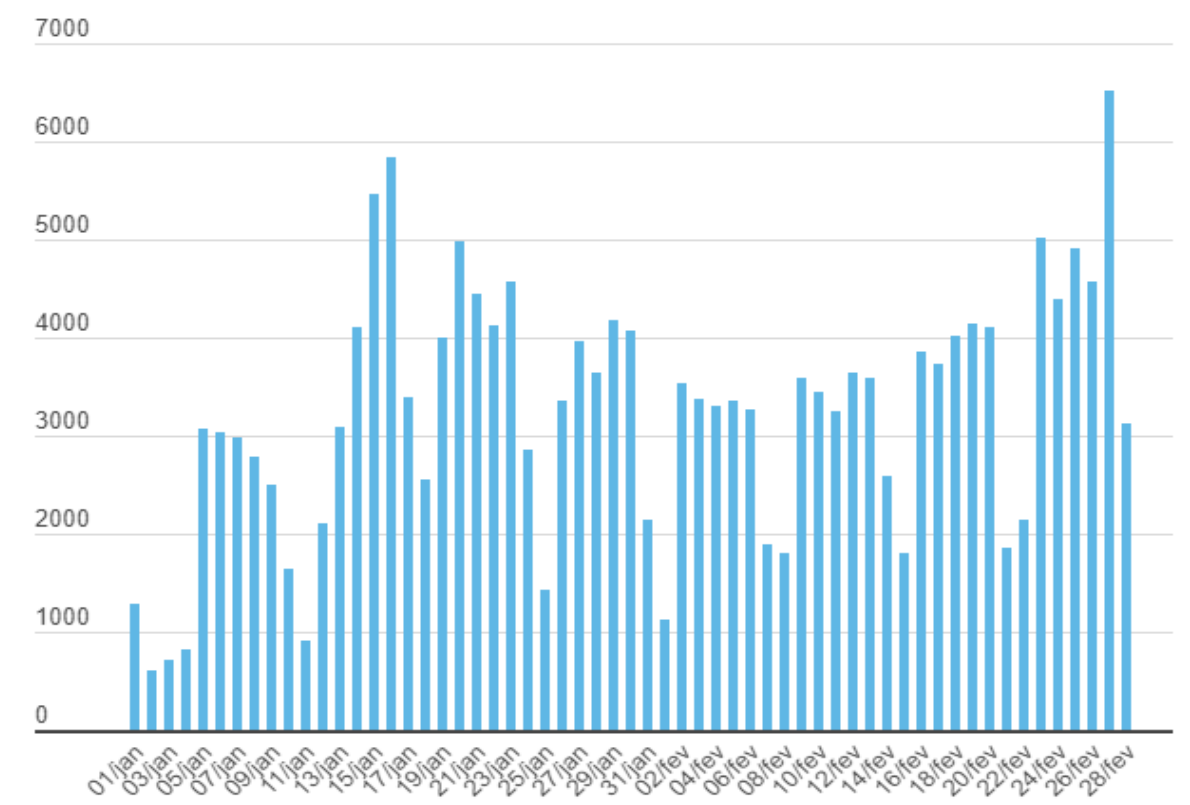

Fonte: Oliveira LR, et al., 2021; dados extraídos de Secretaria Estadual de Saúde da Bahia (SESAB), 2021. 
Gráfico 2 - Número de leitos de UTI ocupados por pacientes com COVID-19 por dia no estado da Bahia.

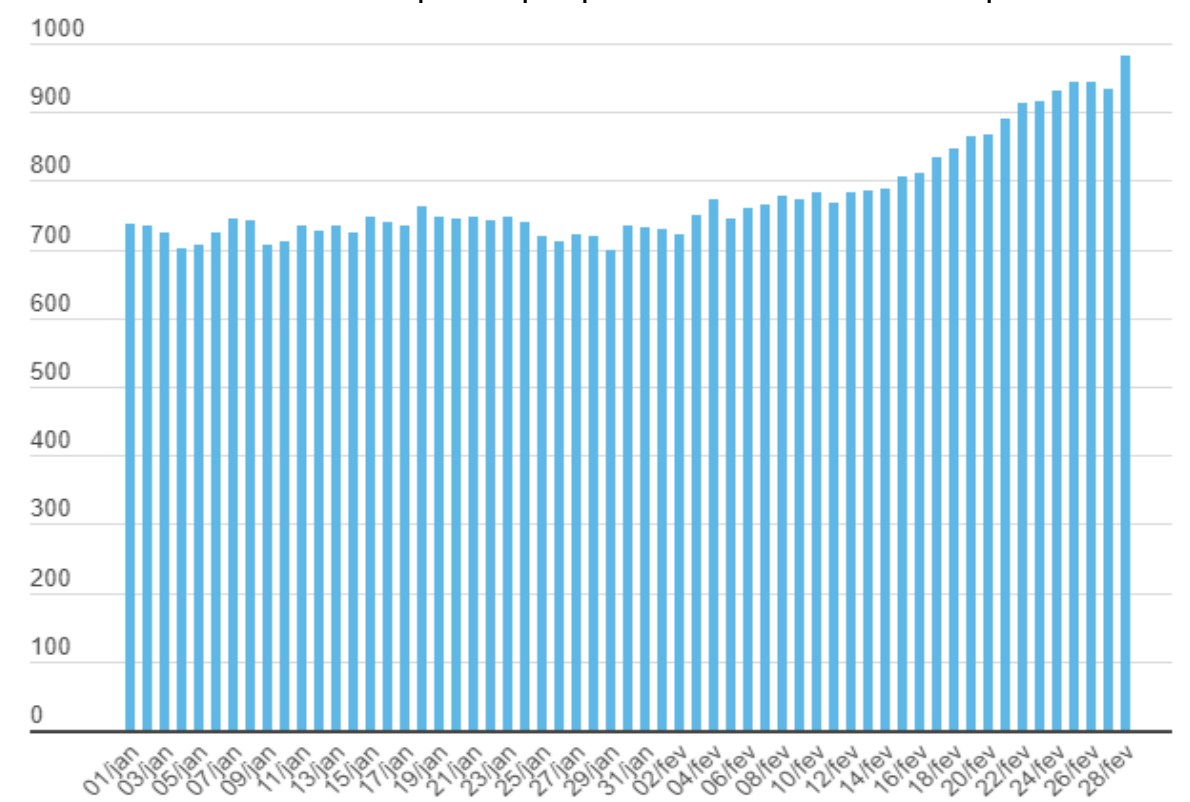

Fonte: Oliveira LR, et al., 2021; dados extraídos de Secretaria Estadual de Saúde da Bahia (SESAB), 2021.

A faixa etária com maior número de casos de COVID-19 na Bahia durante esse período foi a de 30 a 39 anos, com $23,70 \%$ dos casos. A faixa etária de 10 a 19 anos, que representa a maior parcela populacional do estado, ocupa apenas o sexto lugar entre as faixas etárias que mais apresentaram casos, com $6,93 \%$. A Tabela 1 apresenta a quantidade de casos por faixa etária no período e local do estudo.

Tabela 1 - Distribuição de casos de COVID-19 por faixa etária na Bahia entre 01/01/2021 e 28/02/2021.

\begin{tabular}{ccc}
\hline Variável & $\mathbf{N}$ & $\%$ \\
\hline Faixa etária & & \\
\hline$<1$ ano & 1.459 & $0,77 \%$ \\
1 a 4 anos & 2.145 & $1,12 \%$ \\
5 a 9 anos & 2.744 & $1,44 \%$ \\
10 a 19 anos & 13.204 & $6,93 \%$ \\
20 a 29 anos & 34.878 & $18,30 \%$ \\
30 a 39 anos & 45.176 & $23,70 \%$ \\
40 a 49 anos & 37.099 & $19,46 \%$ \\
50 a 59 anos & 25.530 & $13,39 \%$ \\
60 a 69 anos & 15.409 & $8,08 \%$ \\
70 a 79 anos & 8.256 & $4,33 \%$ \\
80 ou mais anos & 4.618 & $2,42 \%$ \\
Ignorada & 119 & $0,06 \%$ \\
\hline Total & $\mathbf{1 9 0 . 6 3 7}$ & $\mathbf{1 0 0} \%$
\end{tabular}

Fonte: Oliveira LR, et al., 2021; dados extraídos de Secretaria Estadual de Saúde da Bahia (SESAB), 2021.

Durante todo o mês de janeiro e fevereiro foram confirmados 2.690 óbitos no estado da Bahia. O Gráfico 3 apresenta a evolução diária durante o período. Do total de óbitos, percebe-se um aumento percentual diretamente proporcional às faixas etárias, com a faixa etária de pacientes com 80 ou mais anos sendo responsável pelo maior número percentual de óbitos (30,30\%), seguidos pela faixa etária de 70 a 79 anos $(25,80 \%)$ e 60 a 69 anos (21,52\%). Além disso, podemos observar que a maior parte dos óbitos ocorridos no estado da Bahia entre janeiro e fevereiro de 2021 são de indivíduos autodeclarados como pardos, sendo responsáveis por 55,69\%, seguido da raça branca $(26,39 \%)$ e preta $(15,09 \%)$. A Tabela 2 apresenta o número absoluto de óbitos por faixa etária e raça autodeclarada e seus respectivos percentuais relacionados. 
Gráfico 3 - Número de óbitos por COVID-19 confirmados por dia no estado da Bahia.

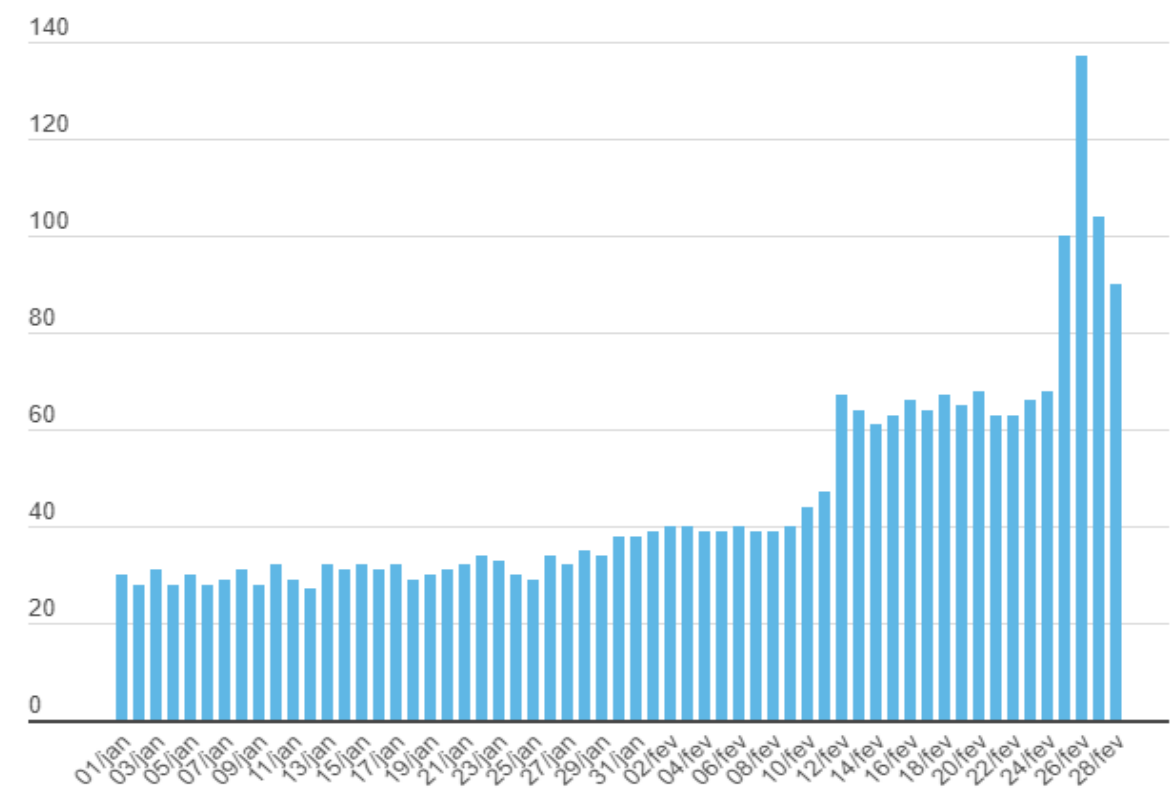

Fonte: Oliveira LR, et al., 2021; dados extraídos de Secretaria Estadual de Saúde da Bahia (SESAB), 2021.

Tabela 2 - Distribuição de óbitos por COVID-19 por faixa etária e raça na Bahia entre 01/01/2021 e 28/02/2021.

\begin{tabular}{ccc}
\hline Variável & $\mathbf{N}$ & $\%$ \\
\hline Faixa etária & & \\
\hline$<1$ ano & 3 & $0,15 \%$ \\
1 a 4 anos & 0 & $0,11 \%$ \\
5 a 9 anos & 11 & $0,41 \%$ \\
10 a 19 anos & 27 & $1,00 \%$ \\
20 a 29 anos & 67 & $2,49 \%$ \\
30 a 39 anos & 170 & $6,32 \%$ \\
40 a 49 anos & 320 & $11,90 \%$ \\
50 a 59 anos & 579 & $21,52 \%$ \\
60 a 69 anos & 694 & $25,80 \%$ \\
70 a 79 anos & 815 & $30,30 \%$ \\
80 ou mais anos & & \\
\hline Raça & 1.498 & $55,69 \%$ \\
\hline Parda & 710 & $26,39 \%$ \\
Branca & 406 & $15,09 \%$ \\
Preta & 4 & $0,15 \%$ \\
Amarela & 5 & $0,19 \%$ \\
Indígena & 67 & $2,49 \%$ \\
Ignorada & $\mathbf{2 . 6 9 0}$ & $\mathbf{1 0 0} \%$ \\
\hline Total &
\end{tabular}

Fonte: Oliveira LR, et al., 2021; dados extraídos de Secretaria Estadual de Saúde da Bahia (SESAB), 2021.

No que diz respeito às comorbidades dos pacientes que vieram a óbito, duas das mais prevalentes na população brasileira figuram como as líderes na estatística: hipertensão arterial sistêmica (33,97\%) e diabetes mellitus $(26,24 \%)$. Isso significa que aproximadamente um a cada três óbitos registrados no estado da Bahia entre janeiro e fevereiro de 2021 possuía hipertensão arterial sistêmica, enquanto um a cada quatro óbitos no mesmo período e local de estudo possuía diabetes mellitus. É válido ressaltar, ainda, que os dados podem se sobrepor, uma vez que o indivíduo pode possuir mais de uma comorbidade. A Tabela 3 apresenta as comorbidades existentes entre os óbitos do período. 
Tabela 3 - Distribuição de óbitos de COVID-19 por comorbidade na Bahia entre 01/01/2021 e 28/02/2021. *

\begin{tabular}{|c|c|c|}
\hline Variável & $\mathbf{N}$ & $\%$ \\
\hline Faixa etária & - & - \\
\hline Hipertensão Arterial Sistêmica (HAS) & 914 & $33,97 \%$ \\
\hline Diabetes mellitus (DM) & 706 & $26,24 \%$ \\
\hline Sem informação & 656 & $24,38 \%$ \\
\hline Doença cardiovascular, exceto HAS & 509 & $18,92 \%$ \\
\hline Sem comorbidades & 181 & $6,72 \%$ \\
\hline Doença renal crônica (DRC) & 147 & $5,46 \%$ \\
\hline Obesidade & 162 & $6,02 \%$ \\
\hline Doença respiratória crônica & 164 & $6,09 \%$ \\
\hline Neoplasias & 130 & $4,83 \%$ \\
\hline Doenças do sistema nervoso & 47 & $1,74 \%$ \\
\hline Demências, incluindo Alzheimer & 68 & $2,52 \%$ \\
\hline Tabagismo & 43 & $1,59 \%$ \\
\hline Imunodeficiências & 35 & $1,30 \%$ \\
\hline Doenças endócrinas, nutricionais e metabólicas, exceto DM e obesidade & 32 & $1,18 \%$ \\
\hline Doença hepática & 16 & $0,59 \%$ \\
\hline Doenças hematológicas & 15 & $0,55 \%$ \\
\hline Outros (glaucoma, osteoartrose) & 32 & $1,18 \%$ \\
\hline Doenças autoimunes & 10 & $0,37 \%$ \\
\hline Doença renal, exceto DRC & 2 & $0,07 \%$ \\
\hline Tuberculose & 2 & $0,07 \%$ \\
\hline Doenças genéticas & 1 & $0,03 \%$ \\
\hline Transtornos mentais e comportamentais devidos ao uso do álcool & 2 & $0,07 \%$ \\
\hline Doença respiratória infecciosa & 0 & $0 \%$ \\
\hline Doenças infecciosas e parasitárias congênitas & 0 & $0 \%$ \\
\hline
\end{tabular}

Legenda: ${ }^{*} E$ válido destacar que os números supracitados não chegam ao valor de $100 \%$ devido ao fato de que um paciente que veio a óbito pode ter mais de uma comorbidade.

Fonte: Oliveira LR, et al., 2021; dados extraídos de Secretaria Estadual de Saúde da Bahia (SESAB), 2021.

\section{DISCUSSÃO}

A sobrecarga do sistema de saúde pública foi a lamentável concretização de uma calamidade anunciada. Diversos estudos culminaram na seguinte direção: se nenhuma ação de ampliação da oferta de leitos e/ou de contenção do vírus fosse realizada, haveria saturação dos sistemas de saúde em um período relativamente curto de tempo (IMPERIAL COLLEGE COVID-19 RESPONSE TEAM, 2020; SARKAR J e CHAKRABARTI P, 2020; VERHAGEN M, et al., 2020; ZHANG T, et al., 2020; PROPUBLICA, 2020).

Tais medidas são necessárias pois, mesmo que uma grande parcela dos indivíduos infectados sejam assintomáticos ou apresentem manifestações leves da doença, uma parcela minoritária de cerca de 15\% evolui com sintomas clínicos mais graves e, destes, $5 \%$ requerem cuidados intensivos e ventilação mecânica, sendo imprescindíveis leitos de UTI (WU Z e MCGOOGAN JM, 2020; PALAMIM CVC e MARSON FAL, 2020).

Somado a isso, a doença possui alta transmissibilidade, desse modo um grande contingente de indivíduos infectados e doentes concomitantemente se traduz por esgotamento da capacidade de atendimento de casos graves e sintomáticos, aumentando de forma significativa os desfechos fatais em decorrência da COVID-19, sobrecarregando o sistema de saúde e, em consequência, inviabilizando tratamento de outras doenças que também necessitam de cuidados intensivos.

Embora no transcorrer da pandemia o estado da Bahia tenha adotado medidas para otimizar os serviços de saúde - principalmente nas cidades com maior população e, por conseguinte, maior número de casos e 
maior sobrecarga no sistema de saúde - como a construção de hospitais de campanha e desburocratização do acesso ao sistema privado de saúde, as medidas não foram suficientes para conter a propagação da COVID-19 (BRASIL, 2020a; BRASIL, 2020b; BRASIL, 2020c).

Diante disso, vê-se como essencial a necessidade de acesso e colaboração do setor privado, posto que no Brasil a maior parte da população recebe o cuidado da Atenção Básica do Sistema Único de Saúde (SUS), enquanto o sistema privado de saúde mantém o maior número de leitos de Unidade de Terapia Intensiva (UTI). Somado a isso, a distribuição de leitos de UTI é desigual entre as regiões do país, comprometendo o atendimento dos pacientes, sobretudo daqueles com sintomas mais severos da doença (ANDRADE CLT, et al., 2020; AQUINO EML, et al., 2020). Desse modo é preciso, em alguns casos, como no contexto pandêmico atual, o Estado recorrer ao setor privado de saúde.

Mesmo com toda mobilização de ampliação de leitos de UTI, realocação de profissionais e compra emergencial de recursos o cenário é preocupante, visto que se traduz no aumento da mortalidade nas localidades onde os serviços de saúde não estão aptos a acompanhar o acréscimo da demanda, principalmente, no atual momento que vem sendo chamado por especialistas como "segunda onda".

À vista disso no Brasil como um todo, apesar das recomendações da OMS, relatórios do Ministério da Saúde e das secretarias estaduais e municipais de saúde, há baixíssima adesão ao uso de equipamentos de proteção individual e isolamento social por parte da população. Em virtude dessa problemática, atualmente, o país tornou-se o epicentro da epidemia na América Latina. Não bastasse isso, a nação ocupa o segundo lugar no mundo em número total de casos, atrás apenas dos Estados Unidos da América (JOHNS HOPKINS, 2021).

Nesse ínterim é importante salientar, ainda, que as medidas de distanciamento social, embora questionadas por parte da população, são de extrema importância para diminuição do número de casos de COVID-19. Aquino EML, et al. (2020) apontam que medidas de distanciamento social, isolamento de casos positivos e seus contactantes apresentam potencial de diminuição da transmissão da doença.

Um outro ponto do estudo que merece destaque é o fato de a população ter passado por datas comemorativas que historicamente são repletas de aglomerações e festividades, de forma melancólica, sem eventos e em isolamento social nas suas casas. Pudemos observar, no entanto, que parte dos brasileiros desrespeitaram tais recomendações, o que reflete nos números. A média de casos na Bahia na segunda semana de janeiro, após as festividades de Réveillon, foi de $2.450,37 \%$ maior que na semana anterior. Isso demonstra o reflexo das aglomerações ocorridas na virada do ano.

A média do número de leitos de UTI ocupados, por sua vez, manteve-se praticamente o mesmo (aumento de 0,34\% na média móvel entre as semanas 1 e 2 do ano de 2021), enquanto a de óbitos teve um discreto aumento de $2,95 \%$ entre as semanas 1 e 2 de janeiro, o que nos parâmetros utilizados indicaria uma estabilidade.

Ao observarmos o Gráfico 1, vemos um aumento do número de casos a partir da segunda quinzena de janeiro, ganhando força no mês de fevereiro. Observa-se que o mês de fevereiro, mesmo apresentando 3 dias a menos que o mês de janeiro, contabilizou mais casos de COVID-19 no estado da Bahia: foram 95.931 casos em fevereiro e 94.706 em janeiro. Isso mostra o aumento exponencial da quantidade de casos, em virtude do relaxamento das medidas de distanciamento social, provocando a "segunda onda".

A gravidade dos casos também aumentou com o avançar da data no período analisado. Com os dados do Gráfico 2 podemos observar que o mês de janeiro apresentou uma média de aproximadamente 730 leitos de UTI ocupados por pacientes com COVID-19 no estado da Bahia, enquanto o segundo mês do ano de 2021 apresentou uma média de cerca de 827 leitos ocupados. Isso representa um aumento de 13,28\% entre os dois meses.

Ao analisarmos o Gráfico 3 vemos que o número de óbitos no estado aumentou consideravelmente nas duas últimas semanas do mês de fevereiro: a média de óbitos dos primeiros 14 dias do mês foi de aproximadamente 45 , enquanto os últimos 14 dias apresentaram uma média de óbitos de cerca de 77 . Isso representa um acréscimo de $71 \%$, o que é extremamente preocupante diante do iminente colapso do sistema de saúde do país como um todo. 
As Tabelas 1 e 2 nos mostram um achado que corrobora com a meta-análise de Hu e Wang (2021) visto que no período e local do presente estudo a maior parte dos casos esteve concentrada nos indivíduos de faixa etária de 30 a 39 anos, todavia, apesar da maior parte do número de casos serem em indivíduos jovens e adultos de meia idade, os mais idosos foram os que tiveram piores desfechos, sobretudo, aqueles sujeitos com 80 anos ou mais. Em vista disso, é consenso na comunidade científica de que é primordial fornecer atenção específica a pacientes idosos, especialmente, aqueles com comorbidades como hipertensão arterial sistêmica e diabetes.

Além disso, a Tabela 2 mostra que os pardos são os que mais morrem, o que é esperado diante da maior parte da nossa população se autodeclarar como parda. A Tabela 3 também nos mostra um dado interessante e consolidado na literatura: indivíduos com comorbidades costumam ter piores desfechos (OLIVEIRA LG, et al., 2021). Apenas $6,72 \%$ dos óbitos ocorridos no período e local do estudo foram atestados como sem comorbidades, enquanto $68,9 \%$ apresentavam pelo menos uma comorbidade. Os dados podem sofrer variações devido ao fato de $24,38 \%$ dos indivíduos que vieram a óbito não apresentarem informações sobre a presença ou ausência de comorbidades.

O acompanhamento da dinâmica epidemiológica da COVID-19 e o seu crescimento exponencial do número de casos nos últimos meses possibilitam a utilização e análise dos dados agregados ao estudo no contexto territorial específico. Somado a isso, permite mapear os padrões de rápida progressão da doença e, assim, predispor meios para subsidiar a tomada de decisão na alocação de recursos para o combate, controle e prevenção em áreas prioritárias do estado da Bahia.

Posteriormente, com os dados deste estudo, poderemos observar a eficácia da vacinação dos indivíduos frente a contenção da pandemia, ao analisarmos se haverá queda do número de casos, ocupação de leitos de UTI e de óbitos. A vacinação contra a COVID-19 iniciou-se no estado da Bahia em 19 de janeiro de 2021, tendo sido vacinadas mais de 430.000 pessoas no período entre a data de início da vacinação e o dia 28 de fevereiro de 2021, em sua grande maioria profissionais de saúde, idosos, indígenas aldeados e idosos que vivem em instituições de longa permanência, pertencentes ao público-alvo da primeira etapa de vacinação. Trata-se de um número muito pequeno frente a necessidade da população, bem como para se atingir a imunidade de rebanho, quando há uma alta taxa de vacinação e, indiretamente, pessoas que não foram vacinadas se beneficiam da aplicação da vacina nos indivíduos que as cercam.

Destacamos também as limitações do estudo, que incluem a utilização de dados secundários relatados pelas secretarias de saúde, tendo em vista que na Bahia, assim como em todo território nacional, não foram implantadas políticas amplas de testagem em massa da população. Dessa forma pressupõe-se que o número de casos COVID-19 no Brasil seja subnotificado.

Uma outra limitação do nosso estudo é que os números aqui apresentados, apesar de serem muito próximos da realidade, não a representam exatamente, principalmente em relação aos óbitos, visto que a Secretaria Estadual de Saúde mantém constante revisão dos óbitos ocorridos no período. Assim, óbitos ocorridos em meses anteriores ao período do estudo podem ter entrado na amostra por terem sido notificados entre janeiro e fevereiro de 2021, bem como óbitos que ocorreram no período do estudo e não foram notificados em tais datas podem ter ficado fora da amostra.

\section{CONCLUSÃO}

Diante das ideias supracitadas neste estudo, verificou-se um aumento substancial dos casos de COVID19 no estado da Bahia, reafirmando o status de segunda onda. Notou-se, também, um crescimento na demanda por leitos de UTI e que os indivíduos mais idosos e com comorbidades possuem piores desfechos. No entanto, observa-se uma mudança desse quadro, com um perfil de pacientes cada vez mais jovens com quadro graves decorrentes da COVID-19. Assim, é fundamental continuar monitorando o comportamento viral e orientando a população para que esta mantenha medidas de isolamento social e uso de máscara. Vale salientar que a vacinação ainda é incipiente e precisaremos de meses para que seja atingida a imunidade de rebanho que nos proporcionará maior segurança e controle da pandemia. 


\section{REFERÊNCIAS}

1. ANDRADE CLT, et al. COVID-19 hospitalizations in Brazil's Unified Health System (SUS). PLOS ONE, 2020: 1-17.

2. AQUINO EML, et al. Medidas de distanciamento social no controle da pandemia de COVID-19: potenciais impactos e desafios no Brasil. Revista Ciência \& Saúde Coletiva, 2020; 25(1): 2423-2446.

3. ARE HOSPITALS NEAR ME READY FOR CORONAVIRUS? HERE ARE NINE DIFFERENT SCENARIOS. 2020. In: ProPublica - Coronavirus. New York: ProPublica. Disponível em: https://projects.propublica.org/graphics/covid-hospitals. Acesso em: 09 mar. 2021.

4. BAHIA. Cidades e Estados. Brasília: Instituto Brasileiro de Geografia e Estatística (IBGE). Disponível em: https://www.ibge.gov.br/cidades-e-estados/ba.html. Acesso em: 07 mar. 2021.

5. BAHIA. Secretaria de Saúde da Bahia. Salvador: Governo da Bahia. Núcleos Regionais de Saúde (NRS). Disponível em: http://www.saude.ba.gov.br/municipios-e-regionalizacao/nucleos-regionais-de-saude-nrs/. Acesso em: 07 mar. 2021.

6. BAHIA. Secretaria de Saúde da Bahia. Salvador: Governo da Bahia. Regiões de Saúde do Estado da Bahia. Disponível em: http://www1.saude.ba.gov.br/mapa_bahia/indexch.asp. Acesso em: 07 mar. 2021.

7. BENNARDO $F$, et al. New therapeutic opportunities for COVID-19 patients with Tocilizumab: Possible correlation of interleukin-6 receptor inhibitors with osteonecrosis of the jaws. Oral Oncology, 2020; 106.

8. BRASIL. BOLETIM EPIDEMIOLÓGICO COVID-19 SVS № 01. 2020. Disponível em: https://portalarquivos2.saude.gov.br/. Acesso em: 13 mar. 2021.

9. BRASIL. LEI № 12.527, DE 18 DE NOVEMBRO DE 2011. 2011. Disponível em:

$\begin{array}{llllll}\text { http://www.planalto.gov.br/ccivil_03/_ato2011-2014/2011/lei//12527.htm. Acesso em: } 07 \text { mar. } 2021 . & \\ \text { 10. BRASIL. LEI NNo 13.979, DE 06 DE FEVEREIRO DE } 2020 . & 2020 . & \text { Disponível em: }\end{array}$ http://www.planalto.gov.br/ccivil 03/ ato2019-2022/2020/lei/l13979.htm. Acesso em: 13 mar. 2021.

11. BRASIL. Plano de Contingência Nacional para Infecção Humana Pelo Novo Coronavírus COVID-19. 2020. Disponível em: https://portalarquivos2.saude.gov.br/. Acesso em: 13 mar. 2021.

12. BRASIL. RESOLUÇÃO № 510, DE 7 DE ABRIL DE 2016 . Disponível em: http://bvsms.saude.gov.br/bvs/saudelegis/cns/2016/res0510 0704 2016.html. Acesso em: 07 mar. 2021.

13. CARSANA L, et al. Pulmonary post-mortem findings in a series of COVID-19 cases from northern Italy: a two-centre descriptive study. The Lancet, 2020; 20(10): 1135-1140.

14. CHEN C, et al. Advances in the research of mechanism and related immunotherapy on the cytokine storm induced by coronavirus disease 2019. Zhonghua Shao Shang Za Zhi, 2020; 36(6): 471-475.

15. CORONAVÍRUS: COMO FORAM CONTROLADAS AS EPIDEMIAS DE SARS E MERS (E NO QUE ELAS SE DIFERENCIAL DA ATUAL). 2020. In: BBC. São Paulo: Arturo Wallace. Disponível em: https://www.bbc.com/portuguese/internacional-52815216. Acesso em: 25 mar. 2021.

16. COVID-19 MAP. 2021. In: Coronavirus Resource Center. Baltimore: Johns Hopkins University \& Medicine. Disponível em: https://coronavirus.jhu.edu/map.html. Acesso em: 25 mar. 2021.

17. DI GENNARO F, et al. Coronavirus Diseases (COVID-19) Current Status and Future Perspectives: A Narrative Review. International Journal of Environmental Research and Public Health, 2020; 17: 1-11.

18. DROSTEN C, et al. Identification of a Novel Coronavirus in Patients with Severe Acute Respiratory Syndrome. The New England Journal of Medicine, 2003; 348(20): 1967-1976.

19. GUAN W, et al. Clinical Characteristics of Coronavirus Disease 2019 in China. The New England Journal of Medicine, 2020; 382: 1708-1720.

20. HU J, WANG Y. The Clinical Characteristics and Risk Factors of Severe COVID-19. Gerontology, 2021: 1-12.

21. IMPERIAL COLLEGE COVID-19 RESPONSE TEAM. Report 12: The Global Impact of COVID-19 and Strategies for Mitigation and Suppression. 2020. Disponível em: https://spiral.imperial.ac.uk:8443/handle/10044/1/77735. Acesso em: 09 mar. 2021.

22. KAI H, KAI M. Interactions of coronaviruses with ACE2, angiotensin II, and RAS inhibitors-lessons from available evidence and insights into COVID-19. Hypertension Research, 2020: 1-7.

23. LEE I, et al. Gastrointestinal and liver manifestations in patients with COVID-19. Journal of the Chinese Medical Association, 2020: 1-3.

24. LU R, et al. Genomic characterisation and epidemiology of 2019 novel coronavirus: implications for virus origins and receptor binding. The Lancet, 2020; 395: 565-74.

25. NOVEL CORONAVIRUS - CHINA. 2020. In: Emergencies Preparedness, Response. Geneva: World Health Organization. Disponível em: www.who.int/csr/don/12-january-2020-novel-coronavirus-china/en/. Acesso em: 04 mar. 2021.

26. OLIVEIRA LG, et al. Análise do índice inicial de casos de Covid-19 relacionado aos indicadores sociais de saúde no estado do Pará, Brasil. Revista Eletrônica Acervo Saúde, 2021; 13(2): 1-11.

27. PALAMIM CVC, MARSON FAL. COVID-19 - The Availability of ICU Beds in Brazil during the Onset of Pandemic. Annals of Global Health, 2020; 86(1): 1-15.

28. ROSE-JOHN S. Interleukin-6 Family Cytokines. Cold Spring Harbor Perspectives in Biology, 2018; 10: 1-17.

29. SARKAR J, CHAKRABARTI P. A Machine Learning Model Reveals Older Age and Delayed Hospitalization as Predictors of Mortality in Patients with COVID-19. MedRxiv, 2020.

30. TURNER AJ, et al. ACE2: from vasopeptidase to SARS virus receptor. TRENDS in Pharmacological Sciences, 2004; 25(6): 291-294.

31. VERHAGEN M, et al. Forecasting spatial, socioeconomic and demographic variation in COVID-19 health care demand in England and Wales. OSF PREPRINTS, 2020: 1-17.

32. WÖLFEL R, et al. Virological assessment of hospitalized patients with COVID-2019. Nature, 2020; 581: 465-469.

33. WORLD HEALTH ORGANIZATION. Report of the WHO-China Joint Mission on Coronavirus Disease 2019 (COVID-19). 2020. Disponível em: https://www.who.int/docs/default-source/coronaviruse/who-china-joint-mission-on-covid-19-finalreport.pdf. Acesso em: 04 mar. 2021.

34. WU X, et al. Co-infection with SARS-CoV-2 and Influenza A Virus in Patient with Pneumonia, China. Emerging Infectious Diseases, 2020; 26(6): 1324-1326.

35. WU Z, MCGOOGAN JM. Characteristics of and Important Lessons From the Coronavirus Disease 2019 (COVID-19) Outbreak in China: Summary of a Report of 72314 Cases From the Chinese Center for Disease Control and Prevention. JAMA, 2020; 323(13): 1239-1242.

36. ZHANG T, et al. A model to estimate bed demand for COVID-19 related hospitalization. MedXriv, 2020: 1-5.

37. ZHOU P, et al. A pneumonia outbreak associated with a new coronavirus of probable bat origin. Nature, 2020; $579(7798)$ : 270-273. 